\title{
What does unification of the Probation Service mean for race equality?
}

Shadae Cazeau examines the unification of probation through the lens of race equality to think about what it means for black, Asian and minority ethnic and Muslim communities in the criminal justice system. 


\section{Introduction - who are EQUAL?}

EQUAL are a National Independent Advisory Group (NIAG) who work collaboratively to improve outcomes for black, Asian and minority ethnic and Muslim communities in the criminal justice system.

EQUAL works collaboratively across the voluntary sector to deliver fair outcomes for all. The NIAG works to support those involved in the development and delivery of criminal justice practice and policy to create a system free from disproportionate outcomes for black and minority ethnic communities.

We believe that action must be taken to address the issues that lead to unjust experiences and outcomes for black and minority ethnic people in the criminal justice system, with one of our key areas of focus being prisons and probation. Having recently reunified it is not yet clear how successful the Probation Service will be in properly engaging and commissioning the voluntary sector, though it is encouraging to see the new commissioning mechanisms and equalities considerations in the Target Operating Model (TOM).

As EQUAL's focus is primarily on race equality, we will consider the implications for ethnic minority staff and service users as well as any opportunities, issues and what we would like to see the NPS committing to moving forward.

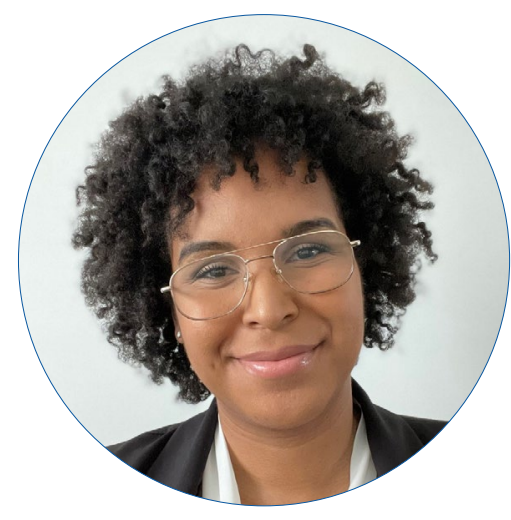

Shadae Cazeau

Head of Policy, EQUAL NIAG

\section{Workforce representation}

Currently, the probation workforce is made up of $10.4 \%$ of people self-declaring as black, Asian or minority ethnic ${ }^{1}$, almost $7 \%$ higher than lower than the proportion of black, Asian and minority ethnic communities across the UK but still disappointing given the disproportionate number of ethnic minorities in the criminal justice system. ${ }^{2}$ However, a recent HM inspectorate of Probation report found that despite staff being recorded nothing has been done with the data to identify and address the disproportionality.

${ }^{1}$ Ministry of Justice. (2020). HM Prison and Probation Service annual staff equalities report 2019 to 2020. https://www.gov.uk/ government/statistics/hm-prison-and-probation-service-annual-staff-equalities-report-2019-to-2020. Accessed 18/08/2021.

2 Office for National Statistics. (2018, updated 2020). Population of England and Wales, from the 2011 Census. 
It is encouraging to see the Probation Workforce Strategy Models has placed a renewed focus on equalities and "creating a more diverse workplace, where everyone feels included" however it is important from our perspective, that ethnicity data is used as a tool to address issues in workforce diversity and not simply as a tick box exercise. $^{3}$

\section{Dynamic framework}

The new dynamic framework and its ability to allow rehabilitative and resettlement services to be commissioned separately to address differing service user needs has possibly been the most significant change as a result of the reunification of the NPS.

This has the potential to be particularly beneficial for minority ethnic service users who need and would benefit from specialist services to address the cultural challenges they experience in rehabilitation and resettlement. However, a report from special interest group RR3 found that despite assurances from Her Majesty's Prison and Probation Service (HMPPS) that qualification for the framework would be simple the process needs to be further simplified and accompanied by guidance. ${ }^{4}$

For the dynamic framework to work effectively it needs to be made accessible so the specialist organisations it seeks to target can be properly considered for commission. This includes ensuring that even once an organisation qualifies onto the framework, they are supported to submit successful bids. We know all too often that smaller, grassroots, and frontline organisations struggle to resource this kind of work in addition to their ongoing service delivery.

\section{Target Operating Model}

The new TOM sets out HMPPS aspirations for the future of probation and commits to focusing on equalities throughout the delivery of the work. This includes equality analysis being undertaken at the beginning and throughout the design of procedures and practices. Crucial to this, is being able to provide evidence of tangible change and where that isn't possible reforming policies and practices under the 'explain or reform' Lammy principle. $^{5}$

The TOM was published in February 2021, before the more recent Police, Crime, Sentencing and Courts Bill began making its way through Parliament. It has therefore been drafted without consideration of the changes introduced by the Bill. The Bill now gives the responsibility for setting licence conditions for fixed term prisoners to probation officers and a new power for a responsible officer to vary a curfew requirement made under a community order or suspended sentence. 
HMPPS will need to consider how they will achieve their equalities objectives when the new and enhanced powers granted by the bill have already been identified as likely to indirectly discriminate against black, Asian and minority ethnic groups. This is even more pertinent considering the recent HM Inspectorate of Probation report: Race equality in probation, which found that three quarters of minority ethnic service users failed to receive referrals or assistance in an area known to affect reoffending (accommodation, mental health etc), that white staff had struggled to understand the backgrounds of service users from minority ethnic groups and those service users' "experiences of racism in probation were spontaneously compared with previous experiences".

Effectively, this means for the TOM to be effective probation staff must become culturally competent, and quickly. Failure to do so will likely undermine the intentions of the TOM and result in further racial disparities as probation powers increase.

Similarly, the same HMIP report found the quality of pre-sentence reports on black, Asian and minority ethnic individuals was insufficient in 21 of the 51 reports inspected and that although initiatives to improve PSR's had been piloted no formal evaluation had been completed. This is a clear cause for concern, especially when the PCSC bill proposes to increase sentences despite identifying in the government's own equality impact assessments that the proposal is likely to indirectly discriminate against minority ethnic groups; making it imperative that PSR's properly reflect the individuals experience and circumstances.

\section{Conclusion}

The aspirations set out by the TOM and the opportunities created by the dynamic framework are admirable, but it is tangible results that are important. The probation workforce should reflect the UK demographic, with probation staff encouraging conversations about race and culture with minority ethnic service users.

Moreover, and most importantly, ethnicity data on outcomes for service users needs to be recorded and published. We know how black, Asian and minority ethnic groups feel about the probation service but without the quantitative data we are unable to really understand or appreciate the true extent of the disproportionality. 